\title{
Salivary Gland Fistula, CTCAE
}

National Cancer Institute

\section{Source}

National Cancer Institute. Salivary Gland Fistula, CTCAE. NCI Thesaurus. Code C57868.

A disorder characterized by an abnormal communication between a salivary gland and another organ or anatomic site. 